\title{
Model Based Planning of Complex Micro-manufacturing Strategies
}

\author{
Daniel Zdebski, Shukri Afazov, Svetan Ratchev, and Joel Segal \\ Precision Manufacturing Centre, The University of Nottingham, University Park, \\ Nottingham, NG7 2RD, UK \\ Daniel.Zdebski@nottingham.ac.uk
}

\begin{abstract}
In recent decades micro-manufacturing becomes increasingly important. Complex multi-material and multi-functional products are required in industries such as bio-medicine, transport, consumer electronics etc. These products, however, cannot be made by single process. Combination of various shaping and assembling processes is required. This paper, gives a systematic overview on the topic of process changing. Clear and systematic process classification system and appropriate design and modelling strategy are presented. Special emphasis is put on planning of complex manufacturing chains. This paper shall help process engineers to make their decisions easier.
\end{abstract}

\section{Introduction}

Our world is changing faster than ever before. The fast trains reach speeds over $500 \mathrm{~km} / \mathrm{h}$; Aeroplanes fly on the boundary of stratosphere, people living over hundred years in good wealth are not exceptional any longer, we have internet connection in any instance nearly anywhere in the world etc. These all would not be possible without rapid scientific development which we have experienced in last decades. One of the research areas promising even more exciting development is micro engineering. Miniaturization of products is essential for better and accessible health care, sensors facilitating safer transport, low energy consumption systems, mobile communication devices and many others.

The micro-manufacturing processes nowadays are capable to produce very complex 3D features with dimensions of few microns and precision in nanometres level. The processes developed recently are capable of machining exotic materials such as shape-memory alloys, zero-thermal expansion materials, explosive materials, hard ceramics and even diamonds. Various micro-manufacturing processes have been developed and extensively researched in research institutions all around the world.

However, most of the micro-products nowadays are manufactured by single manufacturing process or by combination of very few processes. This practice, however, does not allow manufacturing of real multi-functional micro-systems which are still more and more demanded by various industrial fields. Therefore, chaining of various processes seems to be a key for further development of micro-products. However, the problem of processes' chaining is not well reflected in scientific literature. 
Therefore, main scope of this paper is to introduce the problem of chaining of micro-manufacturing processes. New classification system covering all the main stages of micro-manufacturing chain is presented in the first part of this paper. These processes represent main blocks of complex manufacturing chains presented in the second part of this paper. Main logic and method of planning of complex micro-manufacturing strategies are presented. This method helps to develop knowledge based micromanufacturing strategies. It can be used for integration of micro-manufacturing processes as well as for optimization of micro-manufacturing strategies.

\section{Classification of Micro-manufacturing Processes}

Vast number of processes can be used for manufacturing of micro components. For effective choice of right manufacturing process it is essential to understand the fundamentals of different processes. Sensible classification of the processes can be very helpful for further planning of manufacturing strategies. The processes can be classified in various manners. Masuzawa [1] classified the micromachining processes according to the machining phenomena. He divided processes into eight groups; namely: mechanical force, melting/vaporization, ablation, dissolution, plastic deformation, solidification, lamination and recomposition. Alting et. al. [2] has extended Masuzawa's classification by description of the material interactions (subtractive, mass containing, additive and joining). At the other hand, Dimov et. al. [3] classified the processes based on process dimension and feasibility of the processes for machining of different materials. Another possible classification has been presented by Brinksmeier et. al. [4]. Birksmeier divided the micromanufacturing processes between processes originally developed for manufacturing of MEMS (e.g. UV-lithography, silicon micro machining etc.) and micro engineering technologies (micro milling, micro EDM etc.).

Each of the classification systems presented in literature gives some useful information about the processes. However, they all lack the sequence of the processes in the complete manufacturing chain. Therefore, the classification system introduced in this paper divides the processes into four sections representing the main phases of the micro-manufacturing chain. These phases are: 1. Design \& Modelling, 2 . Clamping \&Referencing, 3. Shaping \& Material processing, and 4. Assembly \& System integration.

The whole classification system is illustrated in fig. 1. Obviously the final product quality is affected by each of these phases. Furthermore each phase directly affects the following ones and small errors in the early manufacturing phases can lead to disaster at the end of the manufacturing chain. Therefore, careful planning is fundamental for successful product design.

Processes of each phase classified to groups based on their properties. For example, the processes in shaping \& material processing are classified into three main groups according to material interaction. Three main groups are: subtractive, additive and mass containing processes. Furthermore, the processes are grouped according to their physical similarities. This makes easier to choose right processes. 


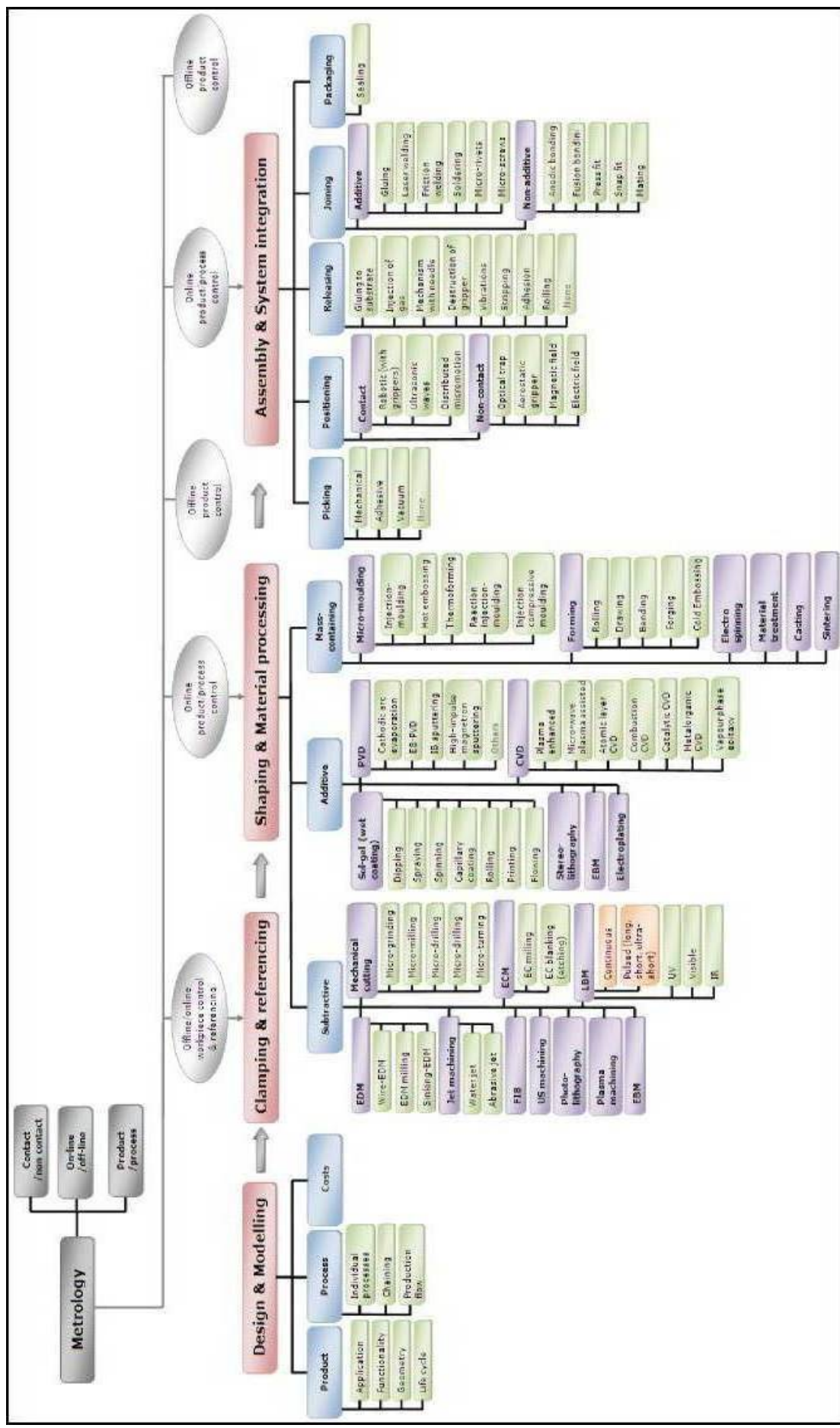

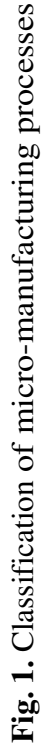


The assembling and packaging processes are then classified in similar manner. In this phase can be identified five main groups of processes. They are: Picking, Positioning, Releasing, Joining and Packaging.

\section{$3 \quad$ Modelling in Micro-manufacturing}

Appropriate modelling helps to increase the manufacturing efficiency and decrease the total costs. It also helps to reduce danger of the project failure. Therefore, it is crucial to proceed with systematic modelling approach covering all aspects affecting micro-manufacturing process.

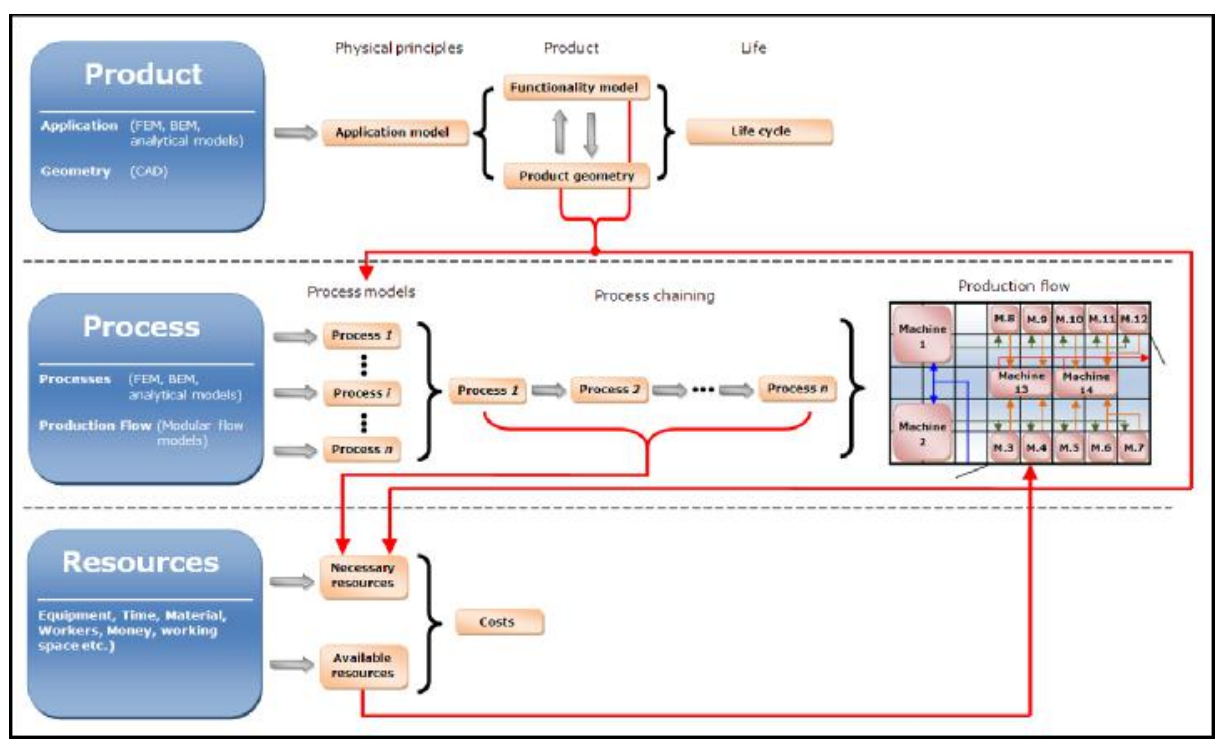

Fig. 2. Strategy for successful design and modelling

The frame of micro-manufacturing modelling is essentially the same as the one for conventional manufacturing. However, due to small dimensions of micro products new challenges arise. The main challenges are: different physical phenomena can be introduced in micro scale, increased level of uncertainties, resolution of the machine tools must be taken in account etc. The fundamental modelling frame is shown in fig. 2. It comprises of three groups representing three main areas of interest. They are: 1) Product, 2) Process and 3) Resources. It is essential for successful planning of manufacturing capabilities and resources to gain comprehensive knowledge on all of these groups.

The product modelling consists of three main phases. In the first phase fundamental physical principles are studied. It is absolutely crucial to have good knowledge about the physical principles responsible for the product functionality. These principles may be different in micro-scale from those known from macro-world. Examples 
may be a capillary effect or effects of van der Walls forces. Majority of models used in this stage are relatively simple analytical or empirical models. However, often it is not possible to apply common macro-models and new models must be developed. This is because of increasing non-linearity due to various effects appearing in micro world.

Once the physical principles are well understood the product geometry can be designed. This must take in consideration limitations of available manufacturing processes, requirements for appropriate product function, assembly of various components, and many others. Therefore, in this stage the engineer has to use more advanced modelling techniques such as FEM, FVD, BEM, CFD, multi-body rigid dynamics and MD. Furthermore, geometric models must be used to study collisions between components and effects of tolerances on the assembly of the product.

Product life is studied in the last step of product modelling. This includes the product wear prediction and fatigue. Furthermore, this stage should also solve issues with products recycling.

The second big group of models solves issues of product manufacturing. This is represented mainly by process chaining and production flow. Both of them help to optimize the production in order to produce the desired possible product with lowest possible resources (time, human work, costs etc.). This group of models represents the most critical stage of the production planning and therefore it further discussed infollowing chapter.

\section{Chaining of Micro-manufacturing Processes}

As stated above the modelling of complex micro-manufacturing chains is not resolved yet. Vella et. al. have proposed an solution of the process chaining through solution of process interactions [5]. However, this approach is not appropriate due to large number of interactions between various processes. Main difficulty is in necessity of solving interactions between individual processes. Furthermore, these interactions are not mutual.

Therefore, in this chapter the main strategy for successful process chaining is presented. The concept of this strategy is based on interchangeable blocks representing individual processes. Each process has many inputs and outputs. For example hardness and surface roughness of EDMed surface depends on workpiece and tool electrical resistivity and thermal conductivity, and process parameters. At the other hand mechanical properties do not influence the output properties of the EDMed surfaceany significantly. Similar examples can be found for all the other manufacturing processes. The relations between various input and output parameters are different for various process. However, some general rules must be kept in order to ensure changeability of the processes. These rules are:

- Each of the process blocks has same input and output parameters,

- The parameters must be same for all processes,

- Models used to solve the links between inputs and outputs must be fast and simple to use,

- The models must take in account process uncertainties, 
General process block is illustrated in fig. 3. Three main groups of inputs and outputs are considered. They are: Information, Material and Energy. Each of these groups contains sequenced data. For example information contains data about shape, surface quality, machining time, costs, product location and many others. In same manner material contains information about material properties such as hardness, stiffness, density, thermal conductivity, electrical resistivity etc. Energy then contains data about energy magnitude, form, stability etc. The links between various inputs and outputs are solved by individual process models.

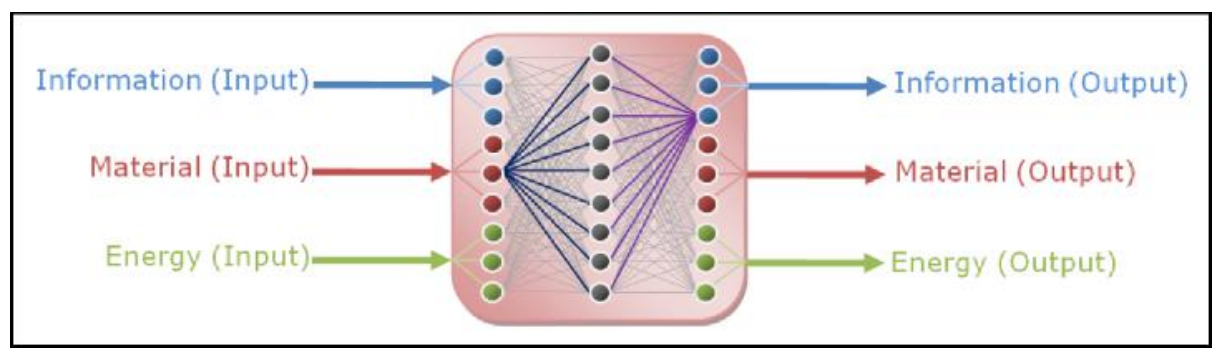

Fig. 3. Illustration of general process

A major issue in micro-manufacturing are high uncertainties. Electrical noise, geometrical tolerances, environmental noise, material impurities has much higher effects on the output parameters in micro scale than in the case of conventional dimensions. D. Zdebski et. al. have presented problem of dimensional tolerances on example of commercial micro end-mills [6]. They found, that typical tolerances of $\varnothing 0.2 \mathrm{~mm}$ micro end-mills represent $15 \%$ of nominal dimensions. This variation of the tools has enormous effect on the tool life and product quality. Therefore, the models used for micro-manufacturing processes must take in account the issue of uncertainties. Especially if complex manufacturing chains are considered the final product uncertainties can be in order of output values. Therefore, all the inputs and outputs should be in form of probability distribution instead of single value (see fig. 4).

Hence, it is clear that this approach requires high number of calculations. Therefore, the models used to solve the links between inputs and outputs should be as fast as possible. Therefore, FEM and other numerically expensive methods are not the right solutions. Simple analytical models are preferable.

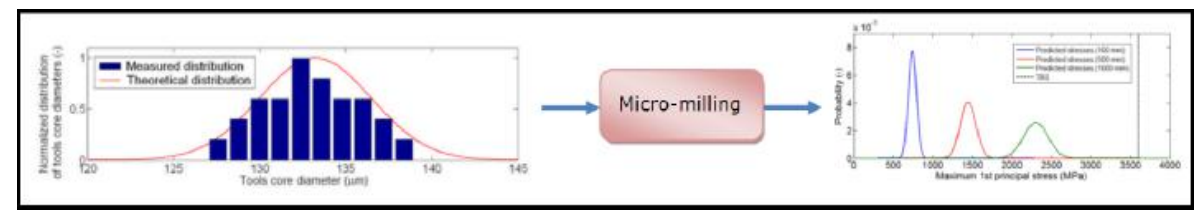

Fig. 4. Illustration of inputs and outputs as a probability distribution function, example of relation of micro end-mill's stresses as a function of tool dimensions 
In fig. 5 is shown a typical process chain. The inputs of the chain are the product requirements and available resources. The requirements can be solve by the approach presented in previous chapter, and they are mainly depended on the product functionality and needs of system integration.

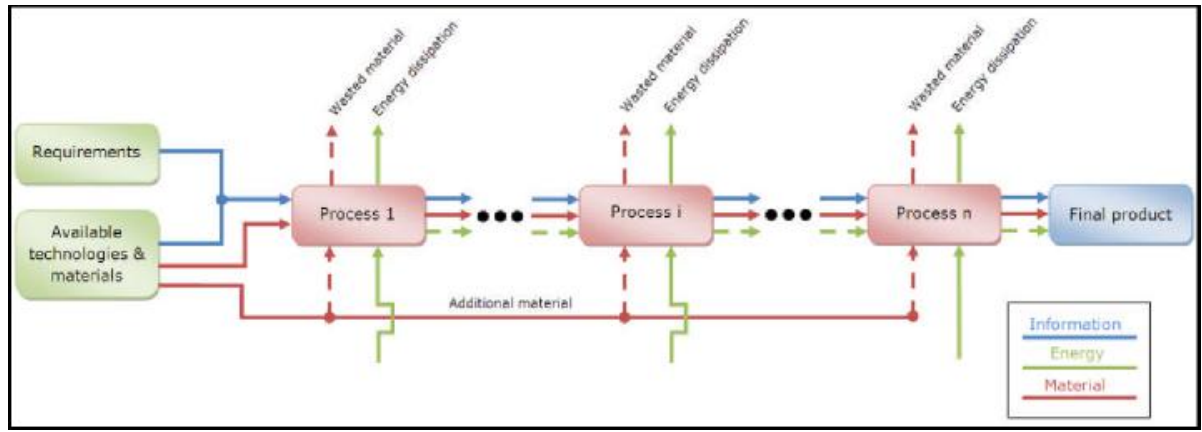

Fig. 5. Example of typical process chain

\section{Example of Micro-pump}

In fig. 6 is shown micro-pump. The overall dimensions of this pump are $500 \mu \mathrm{m} x$ $200 \mu \mathrm{m} \times 200 \mu \mathrm{m}$. Although the presented design does not include motor, valves and packaging, it represents relatively complex product. The product is assembled from 6 individual components. These components are made from different materials (inner cylinder, housing and stopper from aluminium, and stopper and gears from thermoplastic). It is clear that such product cannot be made by single process. At the reality each of the components can be made by various process chains. For example, one possible ways of manufacturing the stopper (one of the simplest components of the assembly) is shown in fig. 7. However, this stopper can be manufactured by other rotes based on additive processes or EDM processes.

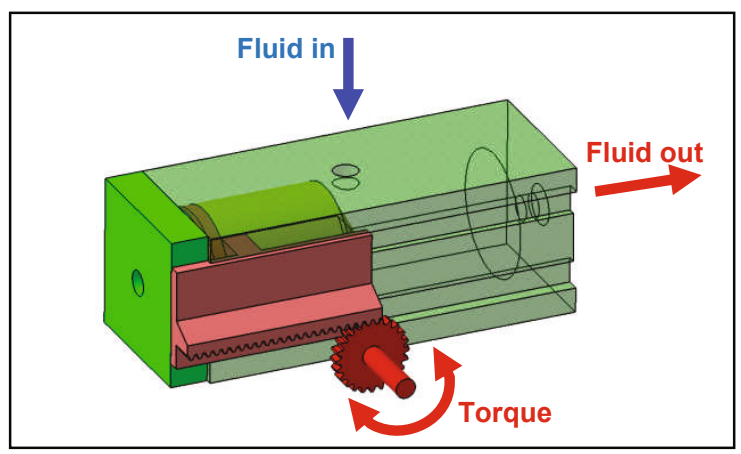

Fig. 6. Micro-pump design

In the case of stopper there is only one critical dimension. However, in other cases there are much more critical surfaces (e.g. linear gear has 7 critical dimensions, and 
inner cylinder and rotational gear have both 5 critical dimensions). Also these components require much higher number of processes. This significantly increases the components' inaccuracies. For example if the rotational gear is manufactured by micro injection-moulding it requires at least 16 processes. Each of these processes affects dimensional errors. Hence, it is that after several processes it is not possible to predict any dimensions without assuming probabilistic approach. This, however, requires good prior knowledge of all individual processes as well as application of robust decision making module, which will help users to choose the most efficient and reliable process chain.

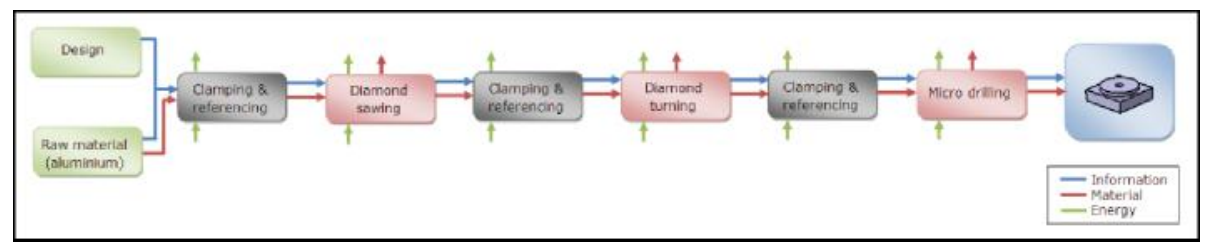

Fig. 7. Process chain for manufacturing of stopper

In fig. 8 is shown an assembly chain for the presented micro-pump. This chain consists of five assembly steps. However, each of these steps contains five substeps: 1) referencing, 2) picking, 3) positioning, 4) releasing and 6) checking. Furthermore, gluing requires an extra substep of application of adhesive material - hence, gluing consists of seven substeps. Hence, only assembling chain consists of minimum of 32 operations. Each of these operations affects final product quality.

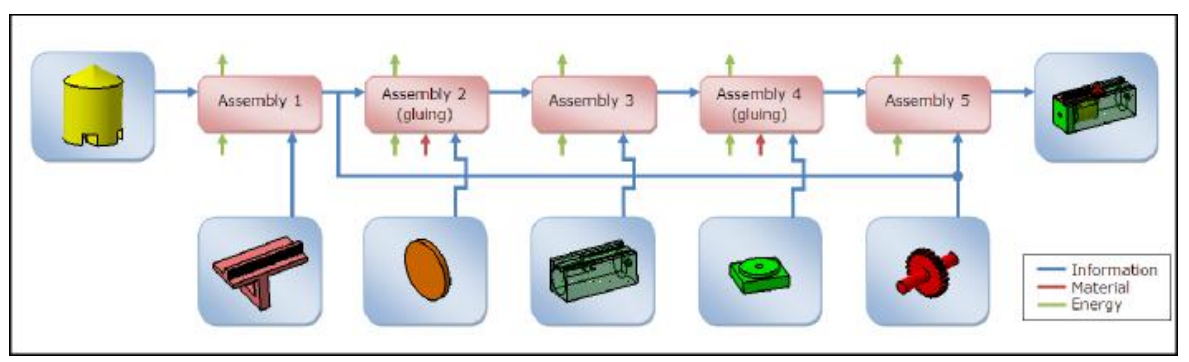

Fig. 8. Assembly chain

\section{Conclusions}

In this paper is presented a strategy for planning complex manufacturing chains. This strategy is based on interchangeable blocks instead of solving interactions between individual processes. The main advantage of this strategy is in its low computational expenses. Another advantage is in possibility of adding arbitrary processes independently on each other. The method is proposed to be used with simple analytical models. However, in contrast with commonly used models, it is proposed to use this method in probability domain. This is because of high effects of process uncertainties during 
micro manufacturing. These uncertainties often accumulate and the cause major problems of assembly of individual components. Also if the probability distribution is used instead of nominal value yield of the process can be estimated.

An example of micro-pump is used to illustrate the process chaining. Whole assembly consist of only six components. However, even this relatively simple product requires minimum of $\sim 150$ operations. Also this product may be manufactured by large number of different process chains.

This paper shows only the main logic of process planning. The future work must be conducted mainly on choice of appropriate models for individual processes and development of robust decision making algorithms. This will lead to development of software which will help to plan micro-manufacturing process chains on analytical (numerical)-statistical models.

Acknowledgements. The authors wish to acknowledge the Nottingham Innovative Manufacturing Research Centre (NIMRC) and the Engineering and Physical Sciences Research Council (EPSRC) for their financial support of the work.

\section{References}

1. Masuzawa, T.: State of the art of micromachining. Annals of CIRP - Manufacturing Technology 49, 473-488 (2000)

2. Alting, L., et al.: Micro Engineering. Annals of CIRP - Manufacturing Technology 52, 635-657 (2003)

3. Dimov, S.S., et al.: Roadmapping study in multi-material micro manufacture. In: Proceedings of the Second International Conference on Multi-Material Micro Manufacture, Grenoble, France, pp. 11-15 (2006)

4. Brinksmeier, E., et al.: Machining of precision parts and microstructures. In Proceedings of the 10th International Conference on Precision Engineering, ICPE, Yokohama, Japan, pp. 3-11 (2001)

5. Vela, P.C., et. al.: A Methodology for Technology Maturity Assessment of Micro and Nano Manufacturing Processes and Process Chains. In: Proceedings of the 4M/ICOMM $2010-$ The Global Conference on Micro Manufacture (2010)

6. Zdebski, D., et al.: A Numerical Analysis of Dimensional Tolerances Effects on the Failure of Micro End-mills. In: Proceedings of the Second International Conference on MultiMaterial Micro Manufacture, Bourg en Bresse, France, pp. 170-173 (2010) 